\section{Sobrevida a mediano plazo en los pacientes con hipertensión arterial pulmonar en la era de terapias vasodilatadoras específicas del territorio vascular pulmonar}

\author{
SEBASTIÁN HERRERA ${ }^{1}$, LUIGI GABRIELLI ${ }^{1}$, ALEJANDRO PAREDES ${ }^{1}$, \\ RODRIGO SAAVEDRA ${ }^{1}$, MARÍA PAZ OCARANZA ${ }^{1}$, \\ PABLO SEPÚLVEDA ${ }^{2}$, HERNÁN DONOSO ${ }^{2}$, LEONEL LÓPEZ ${ }^{2}$, \\ HUGO VERDEJO $^{1}$, FERNANDO BARAONA ${ }^{1}$, PABLO CASTRO ${ }^{1}$
}

\section{Survival of patients with pulmonary arterial hypertension after the advent of specific pulmonary vasodilator therapies}

Background: Pulmonary arterial hypertension $(P A H)$ is a rare and progressive disease. Long-term survival remains poor despite of advances in specific vasodilator therapy. Aim: To describe the survival rate in a cohort of PAH patients in two referral centers in Chile. Patients and Methods: One hundred fifteen patients aged $43 \pm 15.6$ years ( $85 \%$ females) with $P A H$ qualified for this study. Their median pulmonary artery pressure was $55.4 \pm 14 \mathrm{mmHg}$ and their six minutes walking capacity was $368 \pm 119 \mathrm{~m}$. They were followed for $58 \pm 0.4$ months and their actual survival rates were compared with the estimated survival using the equation proposed by the French registry of PAH. Results: One, two and three year survival rates were 97, 94 and 89\%, respectively. The observed survival rates were greater than the estimated survival. Conclusions: The improvement in survival rates observed in this cohort of patients is similar to what has been described in literature.

(Rev Med Chile 2016; 144: 829-836)

Key words: Follow-Up Studies; Hypertension, Pulmonary; Registries; Survival rate.

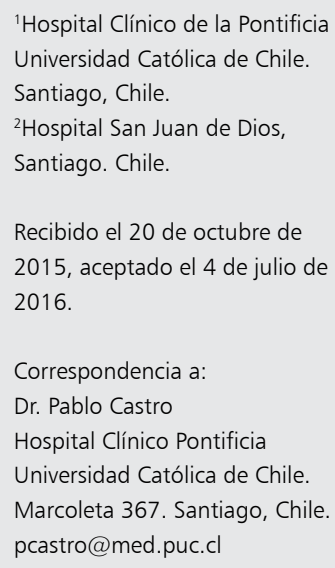

\section{I} a hipertensión arterial pulmonar (HAP) es una condición grave y progresiva, que se caracteriza por cambios proliferativos de los pequeños vasos arteriales pulmonares, lo que produce un incremento de la resistencia vascular pulmonar, con posterior disfunción ventricular y auricular derecha y muerte prematura ${ }^{1}$. Esta condición está caracterizada por la presencia de hipertensión pulmonar pre-capilar y se define por un aumento de la presión de la arteria pulmonar (PAP) media mayor a $25 \mathrm{mmHg}$, en ausencia de aumento de la presión de llenado del ventrículo izquierdo (presión de capilar pulmonar $(\mathrm{PCP}) \leq$ $15 \mathrm{mmHg}$ ).

En Europa, se estima una incidencia anual de 5 a 10 casos por millón de personas; y una prevalencia variable entre 15 y 60 casos por millón de personas ${ }^{2}$, siendo el sexo femenino el más afectado $(80 \% \text { de los casos })^{3}$.

A pesar de los progresos en la terapéutica de la enfermedad, la sobrevida de los pacientes sigue siendo limitada ${ }^{4}$. 
El primer registro que dio información de las características demográficas y el pronóstico de la enfermedad fue el del US National Institutes of Health (1981-1985), donde la sobrevida general (187 pacientes) fue de $35 \%$ a 5 años, siendo la terapia con la que se disponía en aquellos años inefectiva ${ }^{5}$. De este registro se derivó un score de mortalidad, el que actualmente está en desuso, ya que no refleja la realidad pronóstica de la enfermedad en estos tiempos.

En estricto rigor, no es hasta hace 15 años cuando, en base a registros internacionales de Europa y Norteamérica, se han logrado determinar factores de riesgo pronósticos contemporáneos que han permitido caracterizar mejor el comportamiento de la enfermedad. Estos factores han sido de tipos funcionales, demográficos, hemodinámicos y serológicos, ya que se describen que marcadores como el BNP, NT-proBNP o la endotelina 1 tienen un valor pronóstico significativo ${ }^{6}$.

Dentro de los registros más destacados hasta el momento, se puede mencionar el registro norteamericano REVEAL, el registro francés, el registro PHC y el registro de la Clínica Mayo ${ }^{7-10}$.

Respecto de las terapias específicas, se han descrito tres vías principales sobre las que se puede actuar, a saber: 1) La vía del óxido nítrico; 2) La vía de la endotelina y 3) La vía de las prostaglandinas $^{11-13}$. Enríquez publicó, en el año 2011, una serie chilena de 17 pacientes con hipertensión arterial pulmonar pertenecientes al grupo I OMS, que recibiendo terapias específicas mostraron una sobrevida a 1,2 y 3 años de 88,82 y $82 \%$, respectivamente ${ }^{14}$.

El objetivo de este trabajo es reportar la sobrevida actual de los pacientes con hipertensión arterial pulmonar en dos centros especializados del país y compararla con la estimada por una ecuación predictora de riesgo, junto con evaluar el comportamiento de los parámetros de capacidad funcional en el período de seguimiento.

\section{Pacientes y Método}

Se realizó un estudio retrospectivo observacional, aprobado por el comité de ética de las instituciones participantes, en el cual se incluyeron 115 pacientes de los grupos HAP I y IV OMS, controlados en los hospitales San Juan de Dios y Clínico de la Pontificia Universidad Católica de Chile, entre los años 1999 y 2014, los que fueron participantes de estudios internacionales, como, por ejemplo, el estudio SERAPHIN Trial ${ }^{15}$ o el GRIPHON trial ${ }^{16}$. Al momento del diagnóstico se les realizó una evaluación amplia la que incluyó: historia clínica, examen físico, electrocardiograma, ecocardiograma, sondeo cardíaco derecho y test de caminata de $6 \mathrm{~min}$. Además, el estudio se complementó con análisis de los datos demográficos y de laboratorio (pruebas serológicas o de metabolismo). En 56\% de los pacientes se consideró necesario solicitar imágenes pulmonares para descartar la existencia de daño parenquimatoso pulmonar previo o tromboembolismo arterial crónico. HAP fue definida como una PAP media mayor de $25 \mathrm{mmHg}$ y una PCP menor de 15 mmHg. Se consideró como fecha de diagnóstico la correspondiente a la realización del cateterismo cardíaco confirmatorio. Se controló a los pacientes en sus respectivos centros, cada 3 meses; con un seguimiento promedio de 58,5 \pm 43 meses. Se comparó la sobrevida observada con la estimada por la ecuación pronóstica del registro francés ${ }^{17}$ :

$$
\mathrm{H}(t):=\exp (a+b x t)
$$

Donde $a$ y $b$ son parámetros desprendidos del análisis multivariado, y $t$ es tiempo desde el diagnóstico (años). La sobrevida predicha de un paciente en el momento $t$ se obtiene mediante:

$$
\mathrm{P}(t ; x, y, z)=\mathrm{H}(t)^{\mathrm{A}(x, y, z)}
$$

Aquí, A $(x, y, z)$ es una función obtenida del modelo de análisis multivariado, donde " $x$ " es la distancia recorrida en el test de caminata de $6 \mathrm{~min}$ menos 280 metros; " $y$ ” es 1 si el paciente es mujer y 0 si es hombre; y " $z$ " es el gasto cardíaco menos 4 litros por minuto.

Finalmente, la forma funcional de la ecuación es:

$$
\mathrm{A}(x, y, z)=\exp (-(c \mathrm{x} x+d \mathrm{x} y+e \mathrm{x} z)
$$

Donde $c, d$ y $e$ son constantes derivadas del modelo original.

La fecha de fallecimiento fue obtenida mediante búsqueda en la página web del Registro Civil e Identificación de Chile. Para el análisis de los resultados se aplicó estadística descriptiva estándar: las variables cuantitativas fueron descritas utilizando media y desviación estándar y las variables categóricas en forma de porcentaje. Para la comparación de variables continuas se empleó $t$ de Student. 


\section{Resultados}

La edad promedio de los pacientes fue 43,6 $\pm 15,6$ años, con predominio del sexo femenino (85\%). Desde el punto de vista etiológico, 80\% (96 pacientes) pertenecía a HAP del grupo I OMS y $20 \%$ (19 pacientes) a HAP del grupo IV. El síntoma más prevalente en esta serie de pacientes era disnea, la que tuvo un tiempo de evolución hasta el momento del diagnóstico de $17 \pm 9$ meses. $\mathrm{Al}$ inicio del seguimiento, $56 \%$ de los pacientes estaba en clase funcional I-II y $44 \%$ en clase funcional III-IV. El $42 \%$ de los pacientes tuvo alteraciones ecográficas compatibles con disfunción sistólica

Tabla 1. Características basales de los pacientes

\begin{tabular}{|ll|}
\hline Número de pacientes & 115 \\
\hline Relación Hombre:Mujer & $1: 5,3$ \\
\hline Edad (años) & $43,6 \pm 15,6$ \\
\hline $\begin{array}{l}\text { Clase funcional (\%) } \\
\text { I-II }\end{array}$ & $56 \%$ \\
$\quad$ III-IV & $44 \%$ \\
Etiología & \\
$\quad$ Grupo I & $80 \%$ \\
$\quad$ Grupo IV & $20 \%$ \\
\hline Test de marcha 6 min (m) & $368,9 \pm 119$ \\
Valores hemodinámicos & \\
PAPm (mmHg) & $55,4 \pm 14,9$ \\
PAD (mmHg) & $11,9 \pm 7,1$ \\
IC (L/min*m²) & $2,26 \pm 0,62$ \\
RVP (uW) & $13,14 \pm 6,85$ \\
\hline
\end{tabular}

PAPm: presión media de arteria pulmonar; PAD: presión de aurícula derecha; IC: índice cardíaco; RVP: resistencia vascular pulmonar; TAPSE: tricuspid annular plain sistolic excursión. del ventrículo derecho. Se efectuó test de vasorreactividad a $83 \%$ de los pacientes del grupo I OMS, con $9,6 \%$ de positividad.

Un resumen de los pacientes con los valores del cateterismo cardíaco derecho se muestran en la Tabla 1. La angiografía pulmonar es un examen que no se hace de rutina en este tipo de enfermos.

De los 96 pacientes del grupo I OMS, 47 (49\%) tenían el diagnóstico de hipertensión arterial pulmonar idiopática, 45 (47\%) el de hipertensión pulmonar asociada a otras condiciones y $3(4 \%)$ entre hipertensión pulmonar inducida por drogas y la familiar (Figura 1).

Del grupo de HAP asociada a otras condicio-

Tabla 2. Terapias de los pacientes

\begin{tabular}{|lr|}
\hline & \% \\
\hline Terapia convencional & \\
Anticoagulantes & 50 \\
Antagonistas del calcio & 9 \\
Diuréticos & 55 \\
Terapias específicas & \\
Vía de las prostaciclinas & \\
$\quad$ Selexipag (Bloq. receptor PG I $_{2}$ ) & 20 \\
$\quad$ lloprost & 9 \\
$\quad$ Treprostinil & 1 \\
Antagonistas de endotelina & \\
$\quad$ Macitentán & 14 \\
$\quad$ Ambrisentán & 4 \\
$\quad$ Bosentán & 2 \\
Sildenafil & 85 \\
Terapia combinada & $\mathbf{5 0}$ \\
\hline
\end{tabular}

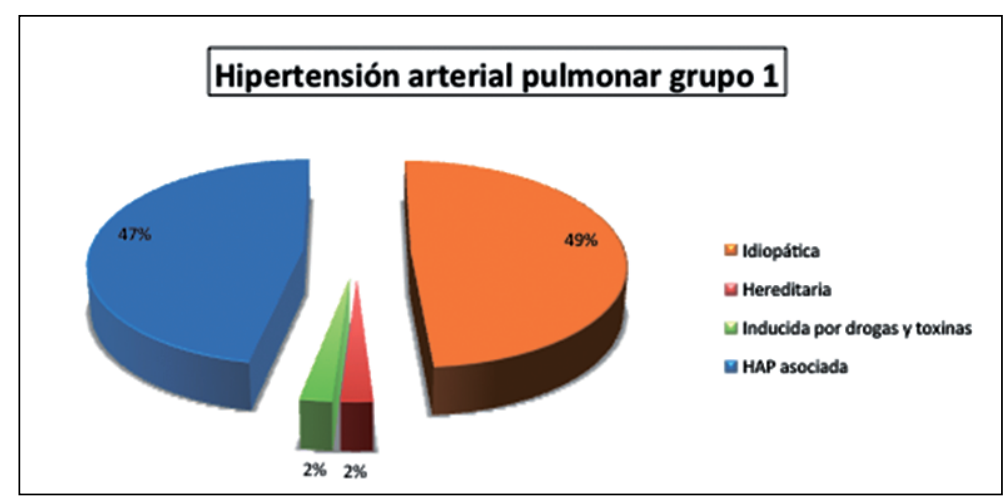

Figura 1. Distribución de los pacientes dentro del grupo $1 \mathrm{OMS}^{7}$. 


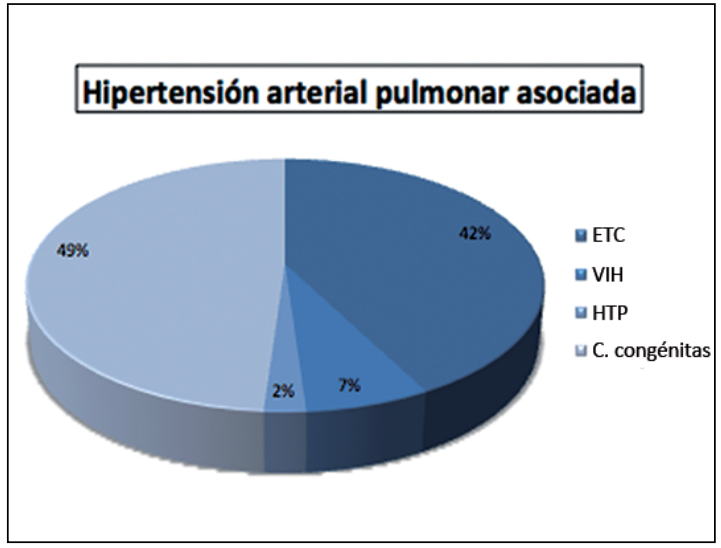

Figura 2. Distribución de los pacientes en la Hipertensión arterial pulmonar asociada a otras condiciones. ETC: enfermedad tejido conectivo; VIH: Virus inmunodeficiencia humana; HTP: hipertensión portal.

nes, 22 (49\%) pacientes tuvieron hipertensión pulmonar asociada a cardiopatía congénita, 19 $(42 \%)$ hipertensión pulmonar secundaria a enfermedad del tejido conectivo, 3 (7\%) hipertensión pulmonar asociada a infección por el VIH y un paciente tuvo hipertensión pulmonar secundaria a hipertensión portal (Figura 2).

\section{Sobrevida}

Durante el período de seguimiento hubo 8 (7\%) fallecidos. Se hizo un análisis de la sobrevida en los pacientes portadores de hipertensión pulmonar arterial idiopática, asociada a fármacos y la familiar, el que arrojó una tasa de sobrevida a 1,2 y 3 años de $97 \%$, 94\% y $89 \%$, respectivamente, la que se comparó con la estimada por la ecuación del registro francés (Figura 3). La sobrevida estimada, según el método de Kaplan Meier, fue de $80 \%$ a los 5 años de seguimiento (Figura 4). Respecto de los tratamientos, $50 \%$ recibió tratamiento anticoagulante oral; $55 \%$ diuréticos y sólo $9 \%$ calcio antagonistas. Dentro de las terapias específicas para controlar la HAP, $85 \%$ de los pacientes recibió sildenafil; $30 \%$, prostanoides y $20 \%$ bloqueadores del receptor de endotelina.

Luego, en las Figuras 5 a 7 se muestran las curvas de sobrevida según etiología, capacidad funcional y tipo de tratamiento: terapia específica única vs terapia específica combinada (sildenafil más un análogo de prostaciclina o un bloqueador del receptor de endotelina) (Figuras 5 y 7 ).

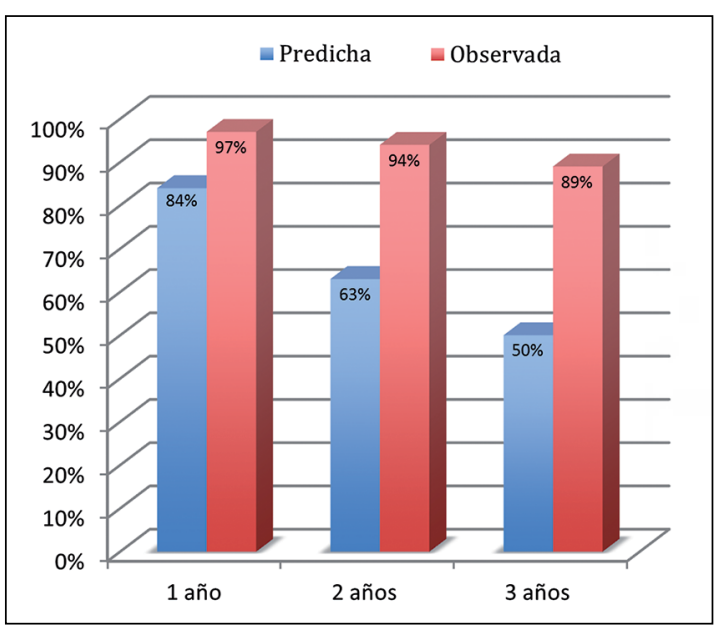

Figura 3. Sobrevida observada y la predicha por la fórmula del registro Francés.

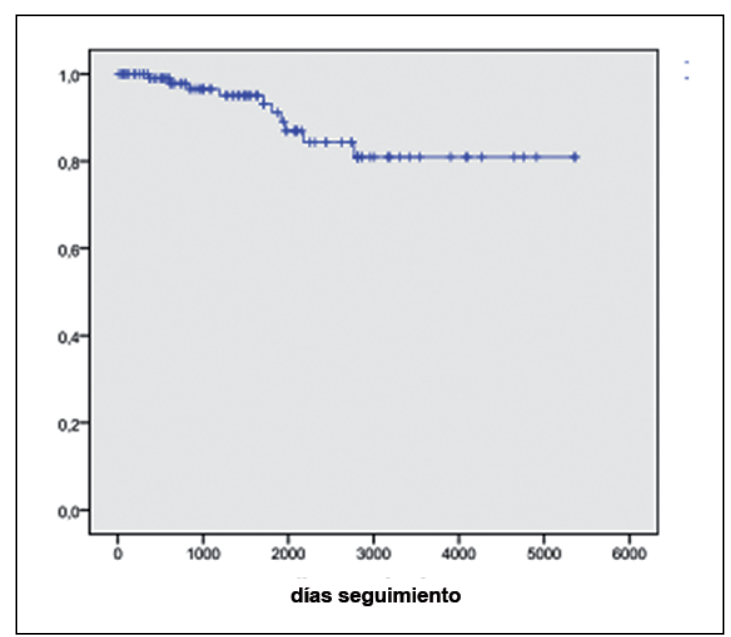

Figura 4. Sobrevida general estimada por Kaplan Meier: $80 \%$ a 5 años.

\section{Parámetros de capacidad funcional}

La distancia promedio recorrida en el test de caminata al momento del diagnóstico fue $368,9 \pm 119$ metros y al año de 386,8 \pm 111 metros $(\mathrm{p}<0,05)$. El índice de disnea de Borg al momento del diagnóstico fue $5 \mathrm{y}$ al año de $3,2(\mathrm{p}<0,02)$. Ninguno de los participantes de esta serie fue sometido a trasplante pulmonar o cardiopulmonar. 

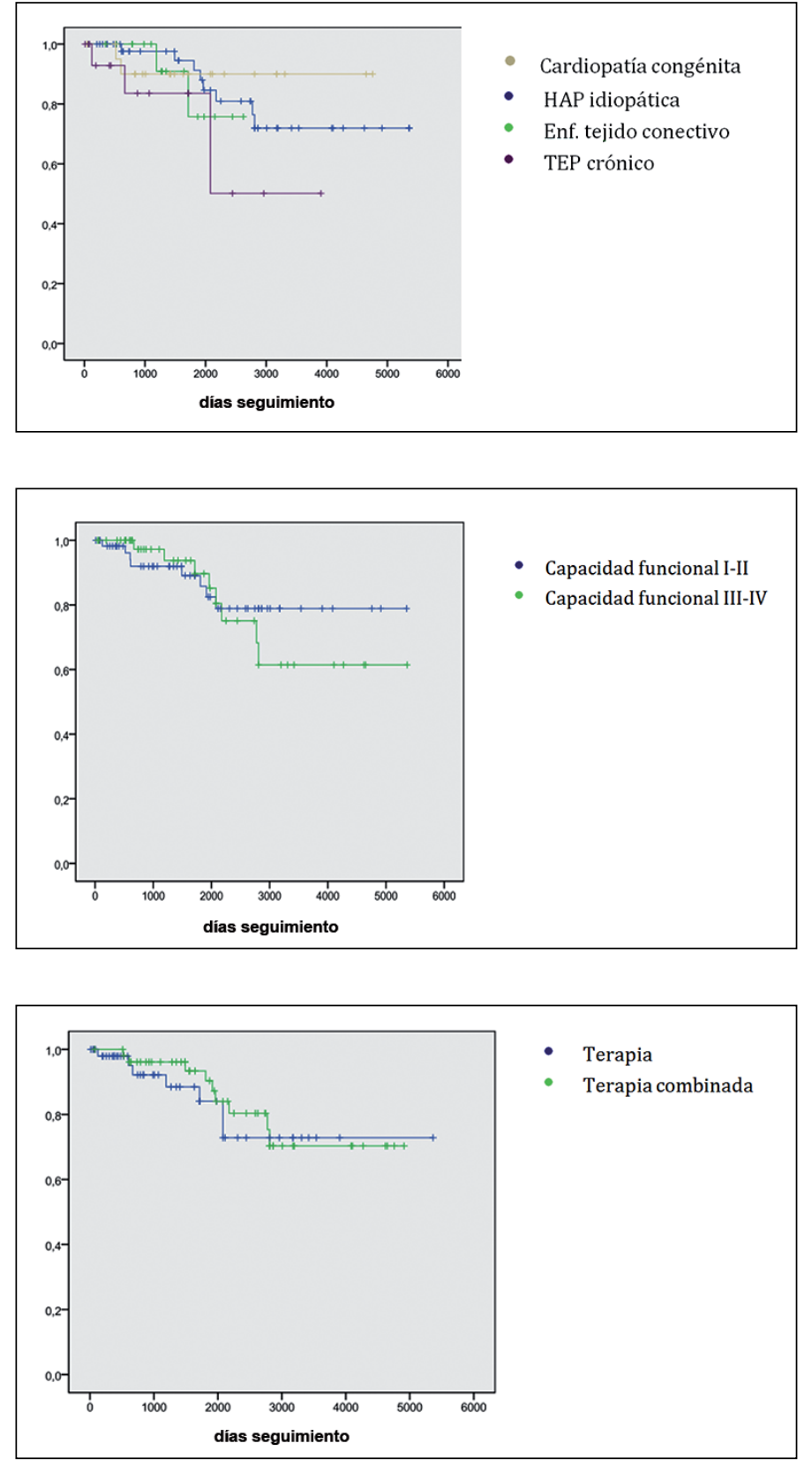

Figura 5. Sobrevida estimada según etiología. Log rank test $\mathrm{p}=0,35$.

Figura 6. Sobrevida estimada según capacidad funcional $(p<0,01)$.

Figura 7. Sobrevida estimada según terapia $(p 0,48)$.

\section{Discusión}

Este trabajo muestra la primera gran serie de pacientes con hipertensión arterial pulmonar a nivel nacional controlados en dos centros de referencia en el manejo de la patología. Consideramos necesario destacar que la sobrevida observada supera sustancialmente a la predicha por una ecuación predictora de riesgo contemporánea y estos resultados son consistentes con los reportados en la literatura reciente. El uso de esta ecuación ha sido sólo validado para su aplicación al grupo de pacientes portadores de HAP idiopática, familiar o inducida por drogas; ya que estas tres variantes son distintos fenotipos de una misma enfermedad. 
En base al registro REVEAL, el más grande publicado hasta el momento, se sabe que la sobrevida mejora cuando las opciones terapéuticas se incrementan y que ésta actualmente es de alrededor de 7 años. Hoy, la edad al momento del diagnóstico es de 50 años, a diferencia de la década 1980-89, cuando el diagnóstico se hacía en promedio a los 35 años de edad. Incluso, la predominancia del sexo femenino ha sido muy variable en los registros recientes, pudiendo no estar presente en edades avanzadas ${ }^{18}$. El registro francés de hipertensión pulmonar se llevó a cabo entre los años 2002 y 2003 , ingresando en él 354 pacientes con hipertensión pulmonar arterial idiopática, familiar y asociada a drogas, estando en NYHA CF III- IV alrededor de $75 \%$ de los pacientes. En este registro, la sobrevida promedio fue de $82 \%$ al año, $67 \%$ a los dos años y de 58\% a los tres años de seguimiento. En el análisis combinado, los predictores independientes de mayor sobrevida fueron: 1) El sexo femenino; 2) Distancia recorrida en el test de marcha y 3) Gasto cardíaco elevado. Se decidió estimar y comparar la sobrevida de la cohorte con la ecuación del registro francés debido a que esta fórmula contiene parámetros clínicos fáciles de obtener en la evaluación inicial de los pacientes. Otras fórmulas, como la del Registro REVEAL, necesitan datos de laboratorio, ej. pro BNP, el que no estuvo disponible en un gran porcentaje de los pacientes.

Los buenos resultados de esta serie chilena pueden estar relacionados con el hecho de que más de la mitad de los pacientes tienen al momento de iniciar el seguimiento una clase funcional I-II, a diferencia de otras series, donde predomina la capacidad funcional III-IV. La edad promedio de diagnóstico estuvo ligeramente bajo el promedio actual, pero muy por sobre la reportada históricamente. Probablemente, la sobrevida de los pacientes de esta serie estuvo determinada por el nivel de atención y manejo otorgado por dos centros especializados en la patología y es evidente que muchos de estos pacientes, al participar de estudios clínicos, tuvieron una atención expedita, pudiendo en ellos pesquisarse precozmente cualquier complicación grave. Por lo demás, la sobrevida también puede estar positivamente influenciada por el hecho que más de la mitad de los pacientes empezó el manejo específico de la enfermedad con grados menores de deterioro de su capacidad funcional.
En nuestro análisis, un determinante importante de la sobrevida en los pacientes fue en qué capacidad funcional se encontraron al momento del ingreso, y esto por sobre el hecho de que hayan sido manejados con monoterapia o terapia combinada específica (Figuras 7 y 8 ).

Cabe destacar que la etiología con mejor sobrevida es la de la hipertensión arterial pulmonar asociada a cardiopatías congénitas (Figura 6).

$\mathrm{Al}$ igual que otras series latinoamericanas ${ }^{19}$, el sildenafil fue la droga específica más usada, a diferencia de los registros europeos ${ }^{20}$ donde los bloqueadores del receptor de endotelina son la primera línea de prescripción. Pese a que sólo 50\% de la población estudiada estuvo bajo tratamiento anticoagulante oral, los resultados de mortalidad no varían sustancialmente respecto de los reportados en otras series, donde los pacientes los reciben mayoritariamente; esto pone nuevamente de manifiesto el dilema de si es adecuado o no indicar a todos los pacientes anticoagulación oral. Hay estudios que mencionan que la existencia de vasorreactividad pulmonar es menos frecuente que lo clásicamente esperado (entre $10-15 \%)^{19}$ y que este hallazgo no constituye un factor protector, lo que actualmente puede estar siendo asumido, ya que en esta base de datos menos de $10 \%$ de los pacientes recibe bloqueadores de los canales del calcio.

Esta es una iniciativa pionera, pero insuficiente, y representa la realidad de sólo dos centros, no pudiendo extrapolarse los resultados a la población entera. Hoy el Estado asumirá, a través de la ley 20.850 "Para el otorgamiento de las prestaciones que cuentan con el sistema de protección financiera para diagnósticos y tratamientos de alto costo", el tratamiento y el seguimiento de los pacientes con hipertensión arterial pulmonar del grupo I OMS, lo que es un avance importante en el manejo de la enfermedad. Como debilidad de este estudio cabe mencionar su carácter retrospectivo, lo que invita a la planificación nacional de un registro que considere tanto a la población prevalente como a la incidente en la enfermedad.

\section{Conclusión}

La hipertensión arterial pulmonar es una patología grave y progresiva que pese a los avances terapéuticos sigue generando un deterioro impor- 
tante de la capacidad funcional en los pacientes y altas tasas de mortalidad. El hallazgo fundamental de este estudio es que la sobrevida observada fue sustancialmente mejor que la estimada mediante una ecuación de riesgo, y que probablemente siga incrementándose a expensas de altos costos en el sistema de salud, tanto en los recursos humanos como en los económicos. En nuestro estudio, no hubo diferencia en la sobrevida de los pacientes en relación a si recibían un fármaco específico versus dos; lo que, creemos no debe ser asumido como tal ya que el " $n$ " del registro es insuficiente para evaluar la efectividad terapéutica de una estrategia farmacológica. En el futuro debemos obtener una ecuación propia para estimar la sobrevida de nuestra población, tomando en cuenta nuestro nivel sociocultural y nuestras influencias ecológicas y genéticas.

\section{Referencias}

1. Haddad F, Spruijt O, Denault A, Mercier O, Brunner N, Furmar D, et al. Right Heart Score for predicting outcome in idiopathic, familial, or drug -and toxin- associated Pulmonary arterialHypertension. JACC: Cardiovascular imaging 2015; 8 (6): 627-38

2. Galiè N, Humbert M, Vachiery JL, Gibbs S, Lang I, Torbicki A, et al. 2015 ESC/ERS Guidelines for the diagnosis and treatment of pulmonary hypertension. European Heart Journal10.1093/eurheartj/ehv317

3. Benza R, Miller D, Gomberg-Maitland M, Frantz R, Foreman A, Coffey Ch, et al. Predicting Survival in Pulmonary Arterial Hypertension: Insights From the Registry to Evaluate Early and Long-Term Pulmonary Arterial Hypertension Disease Management (REVEAL). Circulation 2010; 122: 164-72.

4. McGoon M, Miller D. REVEAL: A contemporary US pulmonary arterial hypertension registry. European Respiratory Review 2012; 123 (21): 8-18.

5. Benza R, Gomberg-Maitland M, Frost A, Frantz R, Humbert M, McGoon M. Development of prognostic tools in pulmonary arterial hypertension:Lessons from modern day registries.Thrombosis Haemostasis 2012; 108: 1049-60.

6. Lador F, Soccal P, Sitbon O. Biomarkers for the prognosis of pulmonary arterial hypertension: Holy grail or flying circus? J Heart Lung Transplant 2014; 33: 341-3.

7. Simonneau G, Gatzoulis M, Adatia I, Celermajer D, Denton C, Ghofrani A et al. Updated Clinical Classification of Pulmonary Hypertension. J Am Coll Cardiol
2013; 62: D34-41.

8. Benza R, Miller D, Foreman A, Frost A, Badesch D, Benton $\mathrm{W}$, et al. Prognostic implications of serial risk score assessments in patients with pulmonary arterial hypertension: A Registry to Evaluate Early and Long-Term Pulmonary Arterial Hypertension Disease Management (REVEAL) analysis. J HeartLungTransplant 2015; 34 (3): 356-61

9. Humbert M, Sitbon O, Chaouat A, Bertocchi M, Habib G, Gressin V, et al. Survival in Patients With Idiopathic, Familial, and Anorexigen-Associated Pulmonary Arterial Hypertension in the Modern Management Era. Circulation 2010; 122: 156-63.

10. Thenappan T, Shan S, Rich S, Tian L, Archer S, Gomberg-Maitland M. Survival in pulmonary arterial hypertension: a reappraisal of the NIH risk stratification equation. Eur Respir J 2010; 35: 1079-87.

11. Kane G, Maradit-Kremers H, Slusser J, Scott Ch, Frantz $\mathrm{R}, \mathrm{McGoon}$ M. Integration of Clinical and Hemodynamic Parameters in the Prediction of Long-term Survival in the patients with pulmonary arterial hypertension. CHEST 2011; 139 (6): 1285-93.

12. Humbert M, Lau E, Montani D, Jaïx X, Sitbon O, Simonneau G. Advances in Therapeutic Interventions for Patients With Pulmonary Arterial Hypertension. Circulation 2014; 130: 2189-208.

13. McLaughlin V, Shah S, Souza R, Humbert M. Management of Pulmonary Arterial Hypertension. J Am Coll Cardiol 2015; 65: 1976-97.

14. Henríquez A, Castro P, Sepúlveda P, Verdejo H, Greig D, Gabrielli L, et al. Cambios en el pronóstico a largo plazo de la hipertensión arterial pulmonar. Rev Med Chile 2011; 139: 327-33.

15. Pulido T, Adzerikho I, Channick R, Delcroix R, Galié N, Ghofrani A, et al. Macitentan and Morbidity and Mortality in Pulmonary Arterial Hypertension.SERAPHIN study.N Engl J Med 2013; 369: 809-18.

16. McLaughlin V, Channick R, Chin K, Frey A, Gaine S, Ghofrani A, et al. Effect of Selexipag on Morbidity/Mortality in Pulmonary Arterial Hypertension. GRIPHON Study. J Am Coll Cardiol 2015; 65 (10_S): doi:10.1016/ S0735-1097(15)61538-8.

17. Humbert M, Sitbon O, Yaïci A, Montani D, O'Callaghan D, Jaïs X, et al on behalf of the French Pulmonary Arterial Hypertension Network. Survival in incident and prevalent cohorts of patients with pulmonary arterial Hypertension. Eur Respir J 2010; 36: 549-55.

18. McGoon M, Benza R, Escribano-Subias P, Jiang X, Miller D, Peacock A, et al. Pulmonary Arterial Hypertension. Epidemiology and Registries. J Am Coll Cardiol 2013; 62: 51-9. 
19. Talavera M, Cáneva J, Favaloro L, Klein F, Boughen R, Bozovich G, et al. Hipertensión arterial pulmonar. Registro de un centro de referencia en Argentina. Revista Americana de Medicina Respiratoria 2014; 2 : 144-52.

20. Mueller-Motte S, Stricker H, Domeninghetti G, Azzola A, Geiser T, Schwerzmann M, et al. Long-Term Data from the Swiss Pulmonary Hypertension Registry. Respiration 2015; 89: 127-40.

21. Jansa P, Jarkovsky J, Al-Hiti H, Popelova J, Ambroz D, Zatocil T, et al. Epidemiology and long-term survival of pulmonary arterial hypertension in the Czech Republic: a retrospective analysis of a nationwide registry. BMC Pulmonary Medicine 2014; 14: 45. 